\title{
Levitation Methods for Structural and Dynamical Studies of Liquids at High Temperatures
}

\author{
I. Egry, D. Holland-Moritz \\ Institut für Materialphysik im Weltraum, Deutsches Zentrum für Luft- und Raumfahrt (DLR), 51170 Köln, Germany
}

\begin{abstract}
In recent years, levitation methods have been increasingly used to study the atomic structure and dynamics of high-temperature liquids, in particular metallic melts. These methods provide a containerless and, consequently, high-purity sample environment. No corrections for signals due to a crucible need to be made, and deep undercoolings of the liquid become possible, reducing the effect of thermal fluctuations. On the other hand, the sample position and, hence, the scattering geometry is not fixed and the free sample surface exhibits capillary waves. Nevertheless, the combination of levitation techniques with x-ray or neutron sources has proven to be possible and successfull. This paper reviews the progress made in this field during the last 10 years or so. It discusses the different levitation techniques: aerodynamic, electromagnetic, electrostatic, as well as the applied spectroscopic techniques: $\mathrm{x}$-ray and neutron diffraction, x-ray absorption and quasi-elastic neutron diffraction. Some recent results are also highlighted.
\end{abstract}

\section{Introduction}

In contrast to solids and gases, liquids remain the least well understood phases of matter. Liquid metals are considered simple liquids in theory, but in practice most are difficult to handle, due to the high temperatures involved. In order to expand our knowledge, and to test the existing recent theories of the liquid state, more experimental information on liquid metal properties and structure is needed.

For metallic melts, containerless processing by different levitation methods are established options. By avoidance of any contact with crucible walls and by providing high purity conditions, the method also allows undercooling of the melt. This non-equilibrium, metastable state is of interest for many reasons: First, a liquid is more quiescent in the undercooled region than in equilibrium, because the temperature is lower by several hundreds of degrees, and, consequently, thermal fluctuations are greatly reduced. This quiescence makes an undercooled liquid suitable for structural studies. Chemical or topological short-range order is certainly more pronounced in the undercooled liquid than in the equilibrium melt. In addition, the fact that an undercooled liquid is not in equilibrium implies that the equilibrium phase diagram does not apply and new, metastable, phases, normally excluded by the phase diagram, may be formed from the undercooled state [1]. This is related to the question how metastability manifests itself in the undercooled liquid, i.e. whether or not there are pre-existing clusters and how, finally, crystallization or vitrification sets in.
Related to the glass transition temperature, $T_{g}$, is the temperature, $T_{\rho}$, at which the densities of the liquid and solid metal become equal. The equality of these two temperatures would imply that the glass transition may not only be characterised as an entropy catastrophe, but, equally well, as a density catastrophe. It is therefore important to measure density and thermal expansion of undercooled liquids [2]. In order to determine the origin of thermal expansion and the extent of anharmonicity it is also necessary to determine the structure of the undercooled liquid or, at least, the temperature dependence of the nearest neighbour distance. Such information, correlated with measurements of thermal expansion, would also reveal the role of free volume in thermal expansion.

The potential for combining containerless processing techniques with synchrotron or neutron sources was recognised and implemented in the 1990's by several research groups worldwide. In the following, a review is given on the activities at DLR. In the next chapter a short introduction is given to the different levitation methods, with particular emphasis on electromagnetic levitation, the workhorse for metallic samples. Consequently, some selected results are presented, including different levitation methods and different structural investigations. 


\section{Levitation Methods}

In order to levitate a massive specimen, the gravitational force must be overcome. This can be achieved by aerodynamic, electrostatic or electromagnetic forces.

Aerodynamic levitation is a technique based on the use of a gas flow for positioning the sample at a stable position. In this method samples are levitated by controlling a gas stream flowing through a nozzle. The levitation force results from the pressure $p$ exerted by a jet of velocity $\mathrm{v}$ in a gas of density $\rho$ :

$p=\frac{\rho v^{2}}{2}$

The conical nozzle levitator [3 ] is the common method to obtain stable aerodynamic levitation of liquids. The sample is supported by a gas flow passing through a diverging conical nozzle. With this method, the sample is usually laser heated from the top. Temperatures above $3000 \mathrm{~K}$ have been obtained with 3 -mm-diameter oxide samples. In the configuration of Hennet et al. [3 ] the cone angle is $60^{\circ}$. In side view, two thirds of the sample can be seen, while the lower third is covered by the cone. The spherical sample is lifted several tenths of a millimeter in an argon flow. For metallic samples, aerodynamic levitation can be combined with inductive heating, a heating coil replacing the laser. Such a hybrid levitator has been built by Mathiak et al. [4] and was used in some of the experiments discussed below.

Electrostatic levitation uses a vertical electric field produced by two condenser plates, to which a high voltage is applied. The sample itself must be charged or polarized, and levitation is achieved through the Coulomb force

$F_{C}=Q E$

where $Q$ is the charge on the sample. Electrostatic levitation is intrinsically unstable, and a fast feed-back mechanism is needed to maintain the sample's position [ ]. The sample must be charged initially, and the charge must be maintained, i.e. loss of charge by evaporation must be compensated by the photoelectric effect. Due to the high voltages applied, electrostatic levitation works best under UHV conditions. In contrast to other levitation methods, the sample is fully visible.

\subsection{Electromagnetic Levitation}

Levitation of electrically conducting samples can be achieved by placing the sample into a high frequency alternating inhomogeneous electromagnetic field, produced by a levitation coil with a conical or cylindrical shape. This field $B$ induces a current in the sample, which in turn, interacts with the field. Levitation is caused by the Lorentz force, $F_{L}$. Its magnitude can be expressed, to lowest order in a multipole expansion, as:

$F_{L}=-\frac{\nabla B^{2}}{2 \mu_{0}} \frac{4 \pi}{3} a^{3} L(q)$ where $\mu_{0}$ is the magnetic permeability, $a$ is the radius of the sample and $q$ is a dimensionless quantity defined as follows:

$q=a \sqrt{\frac{\omega \sigma \mu_{0}}{2}}$

Here, $\sigma$ is the electric conductivity of the sample, and $\omega$ is the frequency of the alternating field. The function $L(q)$ is given by:

$L(q)=\frac{3}{4}\left(1-\frac{3}{2 q} \frac{\sinh (2 q)-\sin (2 q)}{\cosh (2 q)-\cos (2 q)}\right)$

Similarly, the power $P$ absorbed by the sample due to ohmic losses of the induced currents is given by:

$P=\frac{B^{2} \omega}{2 \mu_{0}} \frac{4 \pi}{3} a^{3} H(q)$

with $H(q)$ defined as:

$H(q)=\frac{9}{4 q^{2}}\left(q \frac{\sinh (2 q)+\sin (2 q)}{\cosh (2 q)-\cos (2 q)}-1\right)$

Electromagneic levitation is self-stabilising: a levitated sample is positioned in a potential well generated by the electromagnetic fields. Accordingly, a solid sample is not fixed in position, but performs oscillations about its equilibrium position with a frequency which is determined by the "spring constant" of the field, and its mass. These frequencies are in the range of $5 \mathrm{~Hz}$. In addition, liquid samples exhibit free surface oscillations. The restoring force for these surface oscillations is the surface tension $\gamma$., and the freqiuencies are typically around 50 $\mathrm{Hz}$.

A compact electromagnetic levitator, suitable for the integration into a beamline is shown in Fig. 1. The process chamber is in the center, while the pumps are to the left, and the rf-inductor, connected to the levitation coil through a feedthrough is located to the right. Different viewports for the incoming and scattere beam as well as for the pyrometric temperature measurement, are located at the remaining flanges.

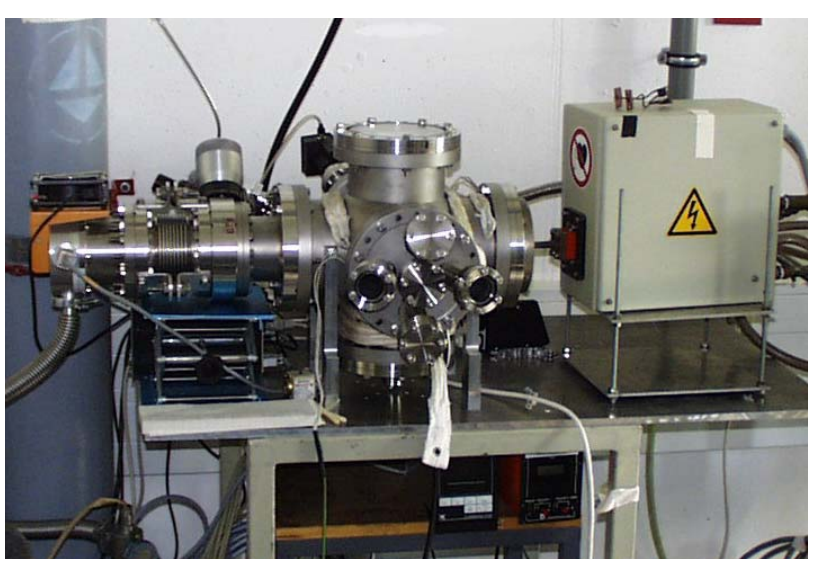

Fig. 1. Electromagnetic levitator with pumps (left), process chamber (center) and rf-inductor (right). 


\section{Results}

\subsection{XAFS, Co-Pd}

If an atom is embedded in a condensed phase, the absorption coefficient is observed to oscillate for energies above the absorption edge. This structure is called XAFS, (X-ray Absorption Fine Structure) and reflects the scattering of the photo-emitted electron by the surrounding atoms.

The XAFS signal is defined as the relative difference between the actual absorption, $\alpha(\mathrm{k})$, and the absorption of an isolated atom, $\alpha(k)$, where $\mathrm{k}$ is the wave vector of the photoelectron:

$$
\chi(k)=\frac{\alpha(k)-\alpha_{0}(k)}{\alpha_{0}(k)} .
$$

A general expression for $\chi(\mathrm{k})$, limited to two-body correlations, is given in ref. [5]:

$\chi_{i}(k)=4 \pi \sum_{j} \rho_{j} \int_{0}^{\infty} g_{i j}(r) \gamma_{i j}^{(2)}(r, k) r^{2} d r$

where $\rho_{j}$ is the number density of species $\mathrm{j}$ and $\mathrm{g}_{\mathrm{ij}}(\mathrm{r})$ is the partial pair distribution function, representing only correlations around the scattering atom of species $i$ with atoms of species $\mathrm{j}$. The function $\gamma^{(2)}(\mathrm{r}, \mathrm{k})$ is the XAFS twobody integral kernel, containing the phase and amplitude of the scattered wave. In modelling $\chi(\mathrm{k})$, a possible asymmetry of the pair distribution function must be taken into account. This is accomplished by using a model for $g(r)$, as proposed by D'Angelo et al. [6]:

$$
\begin{aligned}
g(r)= & \frac{N}{4 \pi \rho r^{2}} \frac{2}{\sigma|\beta| \Gamma\left(\frac{4}{\beta^{2}}\right)}\left(\frac{4}{\beta^{2}}+\frac{2(r-R)}{\sigma \beta}\right)^{\frac{4}{\beta^{2}}-1} \\
& \exp \left(-\frac{4}{\beta^{2}}-\frac{2(r-R)}{\sigma \beta}\right)
\end{aligned}
$$

which depends on the mean atomic distance, $R$, its variance, $\sigma^{2}$, the average coordination number $N$, and the asymmetry parameter $\beta . \Gamma(x)$ is Euler's gamma function. Like in the Gaussian case, $\sigma^{2}$ is the Debye-Waller factor, taking into account both, thermal and statistical disorder. By taking XAFS spectra in fluorescence mode, the absorption $\alpha(\mathrm{k})$, and consequently, the XAFS signal $\chi(\mathrm{k})$, must be corrected for self-absorption according to ref [7] as

$\chi_{0}(k)=\frac{\chi_{\exp }(k)}{1-\Sigma(k)}$

where $\chi_{0}(\mathrm{k})$ is the true signal, $\chi_{\exp }(\mathrm{k})$ the experimentally measured one, and $\Sigma(k)$ the correcting factor for selfabsorption. There are a number of possible strategies to evaluate $\Sigma(k)$ analytically using tabulated values of the absorption cross-section for the various atomic species in the sample, and taking into proper account the sample- detector geometry. We determined $\Sigma(k)$ experimentally, by adjusting XAFS spectra taken in fluorescence mode on levitated solid samples, to XAFS spectra taken in transmission mode on thin solid films of the same composition at room temperature.

The first successful XAFS spectra were taken on a $\mathrm{Co}_{80} \mathrm{Pd}_{20}$ sample at BM29 of ESRF [8]. Spectra were taken over a wide temperature range on both, the Co Kedge, and the Pd L-edge, probing the local environment of the absorbing Co and Pd atom. Nearest neighbour distances, coordination numbers and Debye-Waller factors could be determined. The measured nearest neighbour distance as function of temperature is shown in Fig. 2. Evidently, the temperature dependence is rather weak; on the other hand, the difference in the Co-Co and Co-Pd distances is more pronounced in the liquid than in the solid phase.

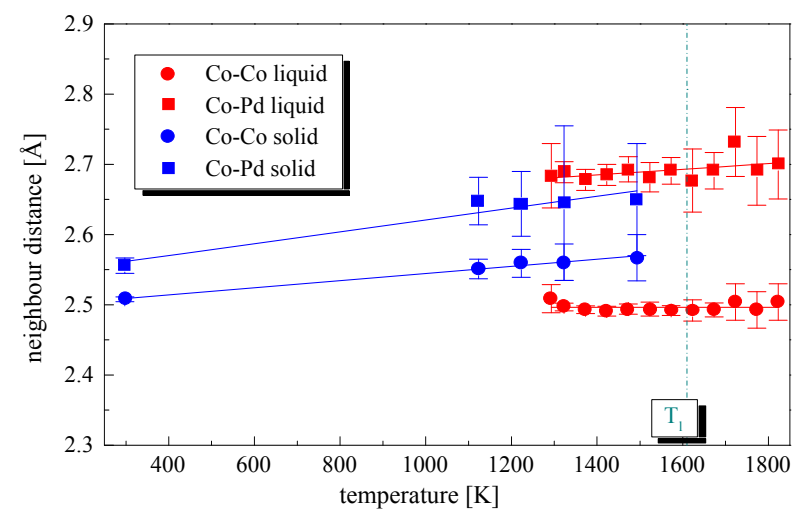

Fig. 2. Neighbour distances in solid and liquid $\mathrm{Co}_{80} \mathrm{Pd}_{20}$ as functions of temperature.

\subsection{EDXRD, Si}

Measurements on undercooled liquids are time-critical. Spontaneous nucleation and subsequent solidification can set in any time. Therefore, experimental techniques with short sampling times are preferred. This is the case for energy dispersive x-ray diffraction (EDXRD), where, in contrast to angle-resolved diffraction, an entire spectrum is recorded instantaneously, without waiting for moving parts to reach their position. The difficulty in EDXRD experiments stems from the background correction. This is achieved by taking subsequently spectra at different angles and fitting them simulataneously by a reverse Monte Carlo technique. This method was applied successfully for determination of the structure factor of pure silicon on ID 09 at ESRF, using electromagnetic levitation [9]. A wide temperature range, including the undercooled regime was covered, and the nearest neighbour distance as well as the coordination number was obtained as function of temperature. This is shown in Fig. 3 and 4, respectively. Our results indicate a negligible temperature dependence of the neighbour distance, and a decrease of the coordination number with increasing temperature, consistent with a positive thermal expansion. Our data are at variance with the findings of Ansell et al. [10] who find that both, the nearest neighbour distance 
and the coordination number increase with temperature. Although further experiments on the short-range order of liquid $\mathrm{Si}$ have been performed $[11,12,13]$ this issue remains controversial.

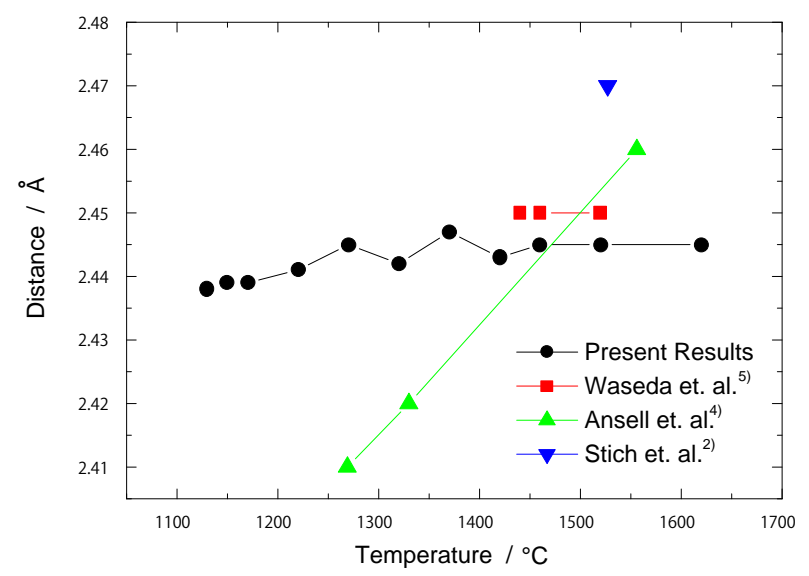

Fig. 3. Nearest neighbour distance in liquid Si. For comparison the results of previous experiments (Waseda, Ansell) and calculation (Stich) is also shown.

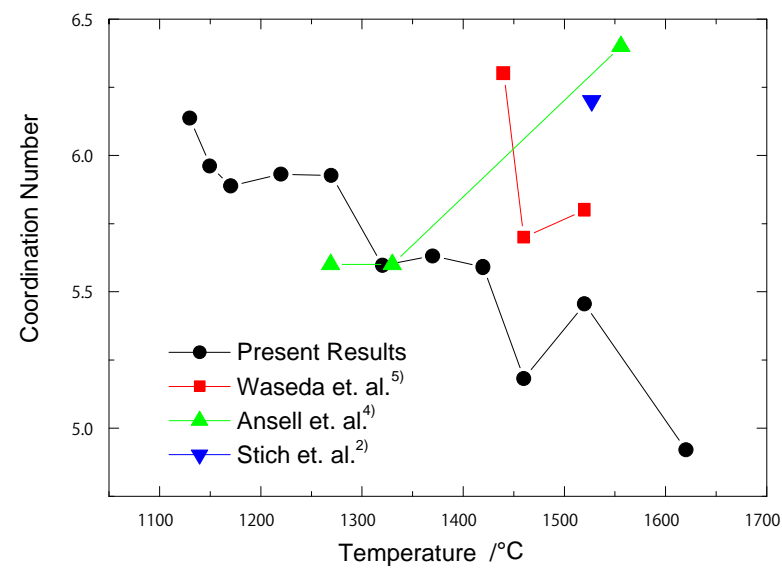

Fig. 4. Coordination number in liquid Si. For comparison the results of previous experiments (Waseda, Ansell) and calculation (Stich) is also shown.

\subsection{HEXRD, AI-Ni}

As pointed out previously, short sampling times are indispensable for experiments on undercooled liquids. As conventional angle-resolved x-ray diffraction, using a goniometer, is slow, it is not well suited for this purpose. On the other hand, the use of an image plate for data acquisition is a perfect alternative: all angles are collected during one exposure, typically of the order of $10 \mathrm{~s}$. By using a high energy beam, a wide q-range can be covered through a small range in angle. The latter is restricted through the gap in the levitation coil. Therefore, High Energy X-ray Diffraction (HEXRD) was applied to study the structure of liquid Al-Ni alloys. Due to the relatively low melting points of these alloys, a hybrid aerodynamicelectromagnetic levitation technique was employed [14].
In particular, alloys with intermetallic $\left(\mathrm{Al}_{3} \mathrm{Ni}\right)$ and eutectic composition (Al-2.7 at $\% \mathrm{Ni}$ ) were studied. For the intermetallic composition, a prepeak in $S(q)$ was found, indicating chemical short-range order. This is shown in Fig. 5.

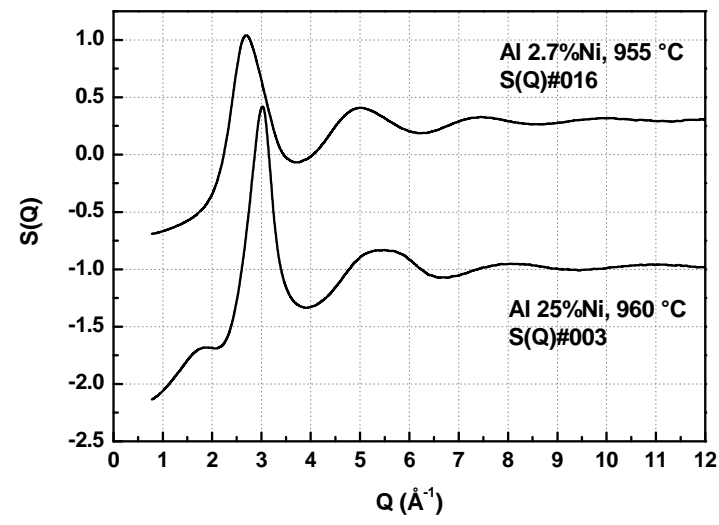

Fig. 5. Structure factor $S(q)$ of liquid Al-Ni alloys.

\subsection{XAFS, AI-Ni}

The diffraction data on liquid Al-Ni alloys indicate the presence of chemical short-range order. However, as this technique is not element-specific, the evidence is rather indirect. In order to support these findings, it is therefore appropriate to complement the diffraction data with results from XAFS measurements, probing the local environment of the nickel atom. Using the same hybrid levitator discussed above, XAFS spectra were taken at BM 29 of ESRF [15]. The spectra were fitted by a two shell model, one for Al, and one for Ni neighbours. As a result of the fitting procedure, the number of nickel neighbours, $n_{N i}$, and the number of aluminum neighbours, $n_{A l}$, was obtained. From these parameters, an order parameter, $\Phi(x)$, can be constructed, where $x$ is the concentration of Ni. It is defined as:

$\Phi(x)=\frac{n_{N i}}{n_{A l}} \frac{1-x}{x}-1$

For a random distribution $\Phi(\mathrm{x})=0 . \Phi(\mathrm{x})>0$ corresponds to homo-atomic association, while $\Phi(\mathrm{x})<0$ indicates a preference for hetero-pairs. The experimentally determined $\Phi(\mathrm{x})$ at $980{ }^{\circ} \mathrm{C}$ is shown in Fig. 6. For all values of $\mathrm{x}, \Phi(\mathrm{x})<0$, indicating a preference for Al-Ni pairs. This behaviour is particularly pronounced at the intermetallic composition $\mathrm{Al}_{3} \mathrm{Ni}$. With increasing temperature, this chemical short-range order is destroyed, as indicated by the star in Fig. 6, obtained at $1150{ }^{\circ} \mathrm{C}$. 


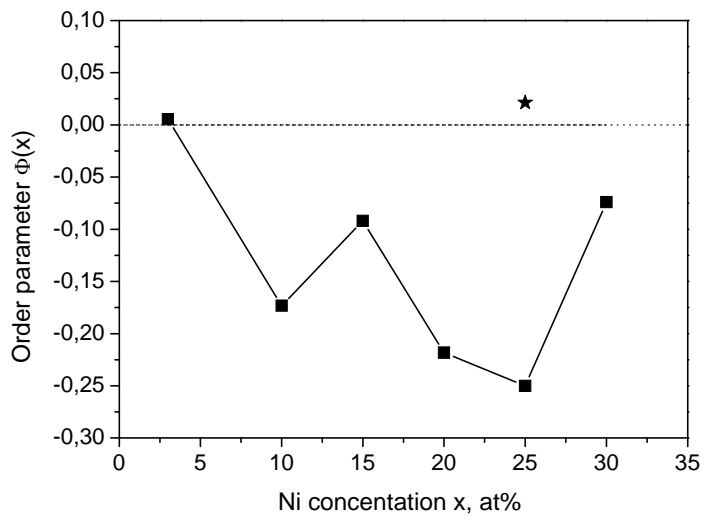

Fig. 6. Order parameter in Al-Ni alloys as function of composition. Full squares correspond to spectra taken at $980{ }^{\circ} \mathrm{C}$, whereas the star refers to a spectrum at $1150{ }^{\circ} \mathrm{C}$.

\subsection{ENS, Transition metals}

In comparison to $\mathrm{x}$-ray experiments, neutron spectroscopy of levitated and undercooled liquid metals presents an even more challenging task. This is due to the fact that the fluxes are smaller by orders of magnitude, and the scattering cross sections are much smaller. This results in very long sampling times, up to hours, during which the sample has to be stably levitated and the temperature kept constant. Nevertheless, Elastic Neutron Scattering (ENS) experiments could be successfully performed at the D20 beamline of ILL, using a dedicated electromagnetic levitation device. In a first experiment, the structure factors of the transition metals $\mathrm{Fe}, \mathrm{Ni}$, and $\mathrm{Zr}$ were determined. In a second step, partial structure factors of an Al-(Co, $\mathrm{Fe})$ were measured.

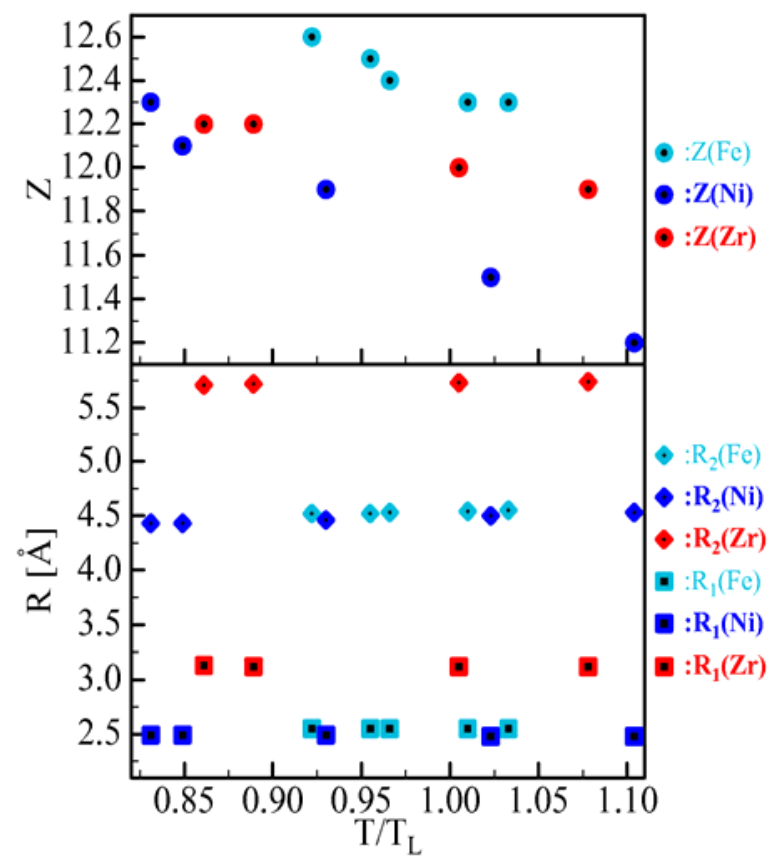

Fig. 7. Coordination number, $Z$, and neighbour distances, R, as function of temperaturef or the three metals $\mathrm{Fe}, \mathrm{Ni}, \mathrm{Zr}$. Temperature is scaled by $\mathrm{T}_{\mathrm{L}}$, the corresponding liquidus temperature.

\subsubsection{Pure metals}

All structure factors $S(q)$ of the three metals Fe, Ni, $\mathrm{Zr}$ showed a characteristic shoulder on the second oscillation, which becomes more pronounced at lower temperatures. The best fit to such behaviour is obtained by assuming an icosahedral order in the melt [16]. From the structure factors, atomic distances and coordination numbers can be derived. This is shown in Fig. 7 as function of temperature. As in the previous cases, the distances are essentially temperature independent, whereas the coordination numbers decrease with increasing temperature.

\subsubsection{Alloys}

In alloys, detailed information about chemical ordering can be obtained from the partial structure factors. Due to the fact that different isotopes of the elements have different cross sections for neutron scattering, partial structure factors can - in principle - be determined by isotopic substitution techniques. If proper isotopes are not available, another option is isomorphous substitution, replacing one element by another, chemically similar, element. The underlying assumption is that the structure remains unchanged. With this method, the partial structure factors of an $\mathrm{Al}_{13}(\mathrm{Co}, \mathrm{Fe})_{4}$ alloy was determined by stepwise replacement of $\mathrm{Co}$ by $\mathrm{Fe}$ [17]. From the experimentally determined partial structure factors, the Bhatia-Thornton pair correlation functions could be constructed, and are shown in Fig.8 for different temperatures. A deep minimum in $\mathrm{G}_{\mathrm{cc}}(\mathrm{r})$ is visible at nearest neighbour distance, indicating chemical shortrange order, and a preference for hetero-atomic pairs. With decreasing temperature, this effect becomes more pronounced.

\subsection{QENS, Ni}

\subsubsection{Ni}

Information about the atomic dynamics can be obtained from the dynamic structure factor $S(q, \omega)$, where $\hbar q$ is the momentum transfer and $\hbar \omega$ is the energy loss. For small energy transfers, in the hydrodynamic limit, the width $\Gamma$ of the quasi-elastic peak of $S(q, \omega)$ is related to the self diffusion coefficient, $D$, of incoherent scatterers through following equation:

$D=\frac{\Gamma}{\hbar q^{2}}$

In order to determine $D$, Quasi-Elastic Neutron Scattering (QENS) experiments must be performed. This is possible at the time-of-flight diffractometer TOF-TOF of the neutron reactor FRM-II in Munich [18]. Monochromatic neutrons are scattered at the levitated sample. The wavevector $\mathrm{q}$ is determined through the angle of the scattered neutron beam, whereas the energy transfer is related to the time of flight between sample and detector bank. First experiments on electromagnetically levitated liquid 
nickel could be successfully performed, and the diffusion constant D could be measured [19]. The obtained dynamic structure factor for a given $q=0.9 A^{-1}$ is shown in Fig. 9 for two different temperatures. From the fit of the data to a Gaussian, the halfwidth $\Gamma$ and, consequently, the diffusion constant $D$ can be obtained. In contrast to macroscopic techniques, this method is free from the influence of convection, due to the different time scales involved.

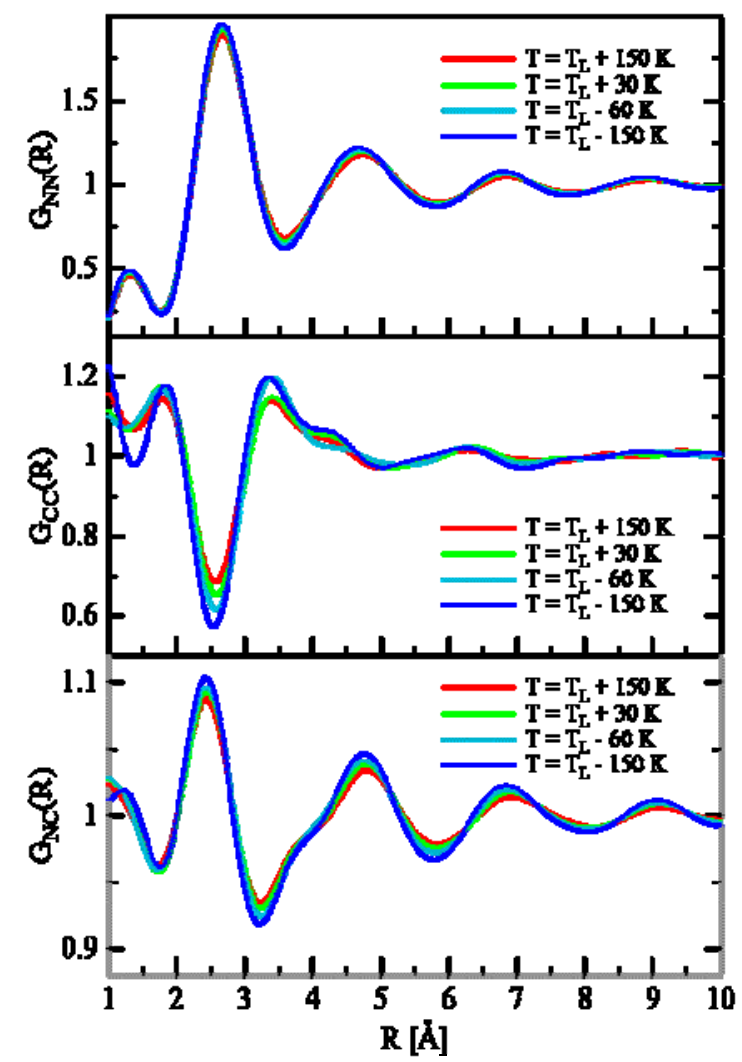

Fig. 8. Bhatia-Thornton partial pair correlation factors for $\mathrm{Al}_{13}(\mathrm{Co}, \mathrm{Fe})_{4}$ for different temperatures obtained by isomorphous substitution.

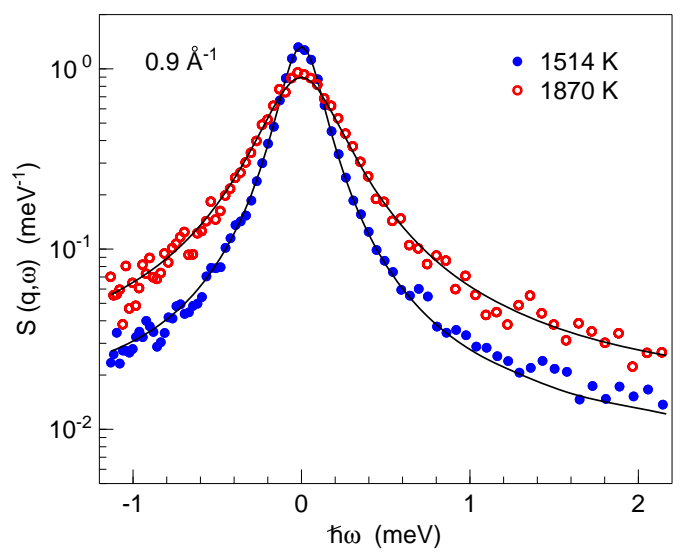

Fig. 9. Dynamic structure factor, $S(q, \omega)$, of liquid $\mathrm{Ni}$ at $q=$ $0.9 \AA^{-1}$ for two temperatures. Open circles correspond to $\mathrm{T}=1870 \mathrm{~K}$, closed circles represent data at $\mathrm{T}=1514 \mathrm{~K}$. .

\subsubsection{Ni-Zr alloys}

Very recently the electrostatic levitation technique has been utilized for quasielastic neutron scattering experiments at the TOF-TOF of FRM-II [20]. By laser heating temperatures in the range between ambient temperature and more than $2500 \mathrm{~K}$ are accessible. Fig. 10 a shows a picture of a freely suspended liquid $\mathrm{Ti}_{39.5} \mathrm{Zr}_{39.5} \mathrm{Ni}_{21}$ droplet with a mass of $0.6 \mathrm{~g}$, processed in the electrostatic levitator at a temperature of $\mathrm{T}=1150 \mathrm{~K}$ and, Fig. 11 shows a raw time-of-flight spectrum measured for a $\mathrm{Zr}_{6} 4 \mathrm{Ni}_{36}$ melt at $\mathrm{T}=1478 \mathrm{~K}$ that was acquired within a range of scattering angles of $59^{\circ}<2 \theta<74^{\circ}$. Also shown is the spectrum of the empty sample environment. Due to the containerless processing setup, the background is about 2 orders of magnitude smaller than the signal measured with sample. Data analysis is still in progress.

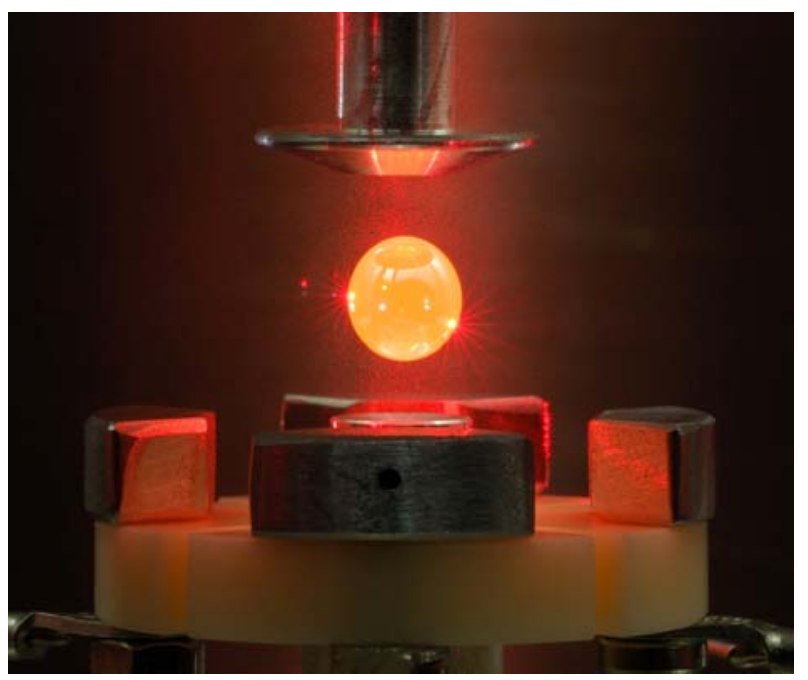

Fig. 10. Electrostatically levitated liquid $\mathrm{Ti}_{39.5} \mathrm{Zr}_{39.5} \mathrm{Ni}_{21}$ droplet with a mass of $0.6 \mathrm{~g}$ at a temperature of $1150 \mathrm{~K}$.

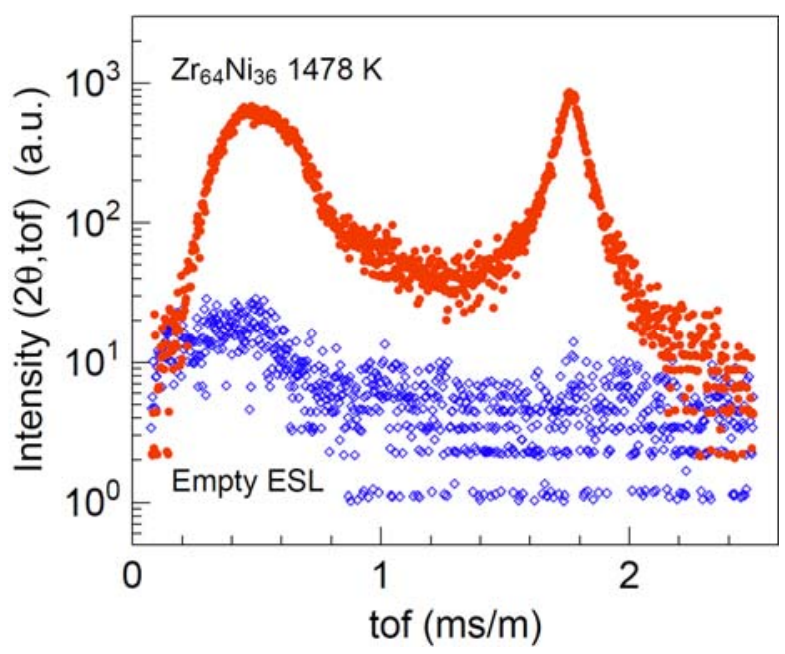

Fig. 11. Raw time-of flight spectrum measured for a $\mathrm{Zr}_{64} \mathrm{Ni}_{36}$ melt at $T=1478 \mathrm{~K}$ in the ESL using a neutron wavelength of $7 \AA$. Also shown is a spectrum of the empty levitator.. 


\section{Summary and Outlook}

In the preceding section we have tried to demonstrate that the combination of levitation techniques with synchrotron and neutron beamlines is a powerful tool, providing information on the atomic structure and dynamics of liquid and undercooled metals which is otherwise hardly accessible.

Generally speaking, atomic distances seem to have only a very weak temperature dependence in all liquid metals investigated, whereas coordination numbers increase as the temperature is lowered, indicating that the liquid structure becomes more perfect. Chemical short-range order is evident in the alloys investigated and seems to be correlated to the phase diagram.

QENS investigations allow to study atomic dynamics in liquids and to determine self diffusion coefficients of incoherently scatterering atoms. Due to the fact that QENS probes the atomic dynamics on atomic length and time scales, this method is unaffected by convective fluid flow that hampers the determination of diffusion coefficients in liquids by classical methods..

\section{References}

1 D. M. Herlach, Mat. Sci. Eng. R12, 177 (1994).

2 L.M. Racz, I. Egry, Rev. Sci. Instrum. 66, 4254 (1995).

3 L. Hennet, D. Thiaudière, M. Gailhanou, C. Landron, J.P. Coutures, D.L. Price, Rev. Sci. Instrum. 73 (2002) 125

4 G. Mathiak, I. Egry, L. Hennet, D. Thiaudiere, I. Pozdnyakova, D. Price, Int. J. Thermophysics 26 (2005), 1151-1166

5 A. Filipponi, J. Phys., Condens. Matter, 13 (2001), R23

6 P. D'Angelo, A. Di Nola, A. Filipponi, N.V. Pavel and P.D. Roccatano, J. Chem. Phys. 100 (1994) 985

$7 \quad$ P. Pfalzer, J.-P. Urbach, M. Klemm, S. Horn, M.L. denBoer, A.I. Frenkel and J.P. Kirkland, Phys. Rev. B 60, (1999) 9335

8 G. Jacobs, I. Egry, Phys. Rev. B, 59, (1999), 3961

9 H. Kimura, M. Watanabe, K. Izumi, T. Hibiya, D. Holland-Moritz, T. Schenk, K.-R. Bauchspieß, S. Schneider, I. Egry, K. Funakoshi, M. Hanfland, Appl. Phys. Letters, 78, 604, (2001)

10 S. Ansell, S. Krishnan, J. J. Felten and D. Price, J. Phys. C11, 8167 (1999)

11 T. H. Kim, G.W. Lee, B. Sieve, A. K. Gangopadhyay, R.W. Hyers, T. J. Rathz, J. R. Rogers, D. S. Robinson, K. F. Kelton, and A. I. Goldman, Phys. Rev. Lett. 95, 085501 (2005).
S. Krishnan, L. Hennet, T. Key, B. Glorieux, M.-L. Saboungi, and D.L. Price, J. Non-Cryst. Sol. 253, 2975 (2007).

13 K. Higuchi, K. Kimura, A. Mizuno, M. Watanabe, Y. Katayama, K. Kuribayashi, Journal of NonCrystalline Solids 3532997 (2007).

14 G. Mathiak, J. Brillo, A. Bytchkov, I. Egry, L. Hennet, I. Pozdnyakova, D. L. Price, D. Thiaudiere, D. Zanghi, J. Non-Cryst. Solids, 352 (2006), 4008

15 I. Egry, L. Hennet, M. Kehr, G. Mathiak, S. De Panfilis, I. Pozdnyakova and D. Zanghi, J. Chem. Phys., 129, (2008), 064508, 1-5

16 T. Schenk, D. Holland-Moritz, V. Simonet, R. Bellissent and D.M. Herlach, Phys. Rev. Lett., 89, 075507 (2002).

17 T. Schenk, V. Simonet, D. Holland-Moritz, R. Bellissent, T. Hansen, P. Convert, D.M. Herlach Europhys. Lett. 65, 34 (2004).

18 T. Unruh, J. Neuhaus, and W. Petry, Nucl. Instrum. Methods Phys. Res. A 580, 1414 (2007)

19 A. Meyer, S. Stüber, D. Holland-Moritz, O. Heinen, T. Unruh, , PRB 77 (2008) 092201

20 T. Kordel, D. Holland-Moritz, T. Unruh, J. Peters, A. Meyer, to be published 